\title{
Anti-EGFR antibody conjugated silica nanoparticles as probes for lung cancer detection
}

\author{
JUN WAN ${ }^{1}$, WEI WU ${ }^{2}$, RENQUAN ZHANG ${ }^{3}$, SHANDONG LIU $^{3}$ and YUNLONG HUANG ${ }^{3}$ \\ ${ }^{1}$ Department of Thoracic Surgery, The Shenzhen People's Hospital, The Second Clinical Medicine College \\ of Jinan University, Shenzhen, Guangdong 518020; Departments of ${ }^{2}$ Hematology and ${ }^{3}$ Thoracic Surgery, \\ The First Affiliated Hospital of Anhui Medical University, Hefei, Anhui 230022, P.R. China
}

Received March 16, 2016; Accepted March 3, 2017

DOI: $10.3892 / \mathrm{etm} .2017 .4988$

\begin{abstract}
A well-designed nanosystem [anti-epidermal growth factor receptor-MB-encapsulated thiol-terminated silica nanoparticles (EGFR/MB-SHSi) complexes] containing silica nanoparticles and near-infrared fluorescence dye (NIRF) methylene blue (MB) was established as a tumor-targeted probe for potential lung cancer detection. The anti-EGFR/MB-SHSi complexes exhibited desirable and homogenous particle size, high bovine serum albumin stability, low hemolytic activity, neutral surface charges and negligible cytotoxicity in vitro. Furthermore, the results of confocal laser scanning microscopy and flow cytometry confirmed that the EGFR-targeted function induced high and specific cellular uptake of anti-EGFR/MB-SHSi complexes. In vivo investigation of nude mice bearing A549 tumor xenografts revealed that anti-EGFR/MB-SHSi complexes possessed strong tumor target ability. These observations indicated that anti-EGFR/MB-SHSi complexes may be a safe and tumor-targeting probe for the detection of cancer.
\end{abstract}

\section{Introduction}

Lung cancer is one of the most lethal types of cancer and the second most common cancer in both women and men worldwide with a staggering $28 \%$ of the total cancer-associated mortality rate in the United States alone $(1,2)$. Early detection of cancer is critical and is expected to contribute significantly to the success of cancer therapy and to the improvement of patients' survival rates (3). Additionally, fluorescence imaging, one of the optical imaging approaches, is a desirable modality for the early detection of cancer due to its multiplex detection

Correspondence to: Dr Jun Wan, Department of Thoracic Surgery, The Shenzhen People's Hospital, The Second Clinical Medicine College of Jinan University, 1017 DongMen North Road, Shenzhen, Guangdong 518020, P.R. China

E-mail: junwan1976@sina.com

Key words: epidermal growth factor receptor, lung cancer detection, silica nanoparticles abilities and high sensitivity, which depends on the probes emitting in the near-infrared fluorescence (NIRF) spectrum window (wavelength, 650-900 nm) (4). However, the application of NIRF as a probe in cancer detection remains difficult because conventional NIRF organic dyes have many disadvantages, including insufficient stability in biological systems and low detection sensitivity (5).

Nanotechnology has engendered a range of novel materials with unique properties, unlike their bulky counterparts, suggesting that these difficulties may be overcome to ensure effective delivery of NIRF dyes $(6,7)$. Furthermore, numerous uses of nanoparticles, including micelles, polymer nanoparticles, liposomes, iron oxide nanoparticles and silica nanoparticles have been reported for NIRF dye delivery (8-12). Such nanostructured materials may protect the NIRF dyes from environmental degradation and at the same time greatly increase dye loading and uptake into tumor sites and reduce nonspecific damage to normal tissues over free photosensitizers by means of the 'enhanced permeability and retention effect' (13). Furthermore, these advantages can be further enhanced by modifying the nanoparticles with certain targeting ligands $(13,14)$.

Methylene blue (MB) is considered to be one of the most inexpensive commercially available NIRF probes and has been widely used in the field of bioanalysis (15). However, its application in cancer detection is limited largely due to its molecular structure as it lacks reactive functional groups is highly hydrophilicity, which makes it difficult to conjugate or effectively incorporate MB into conventional nanoparticles, such as micelles and liposomes (16). Previously, silica nanoparticles have emerged as a preferable carrier. Compared with polycations, there is little concern about their toxicity. In contrast to liposomes, the inorganic nanoparticles should be resistant to lipases and bile salts encountered in the gastrointestinal tract, physical stress during aerosolization and should withstand autoclaving, in principle (17). In addition, the silica nanoparticles can also be easily tailored to meet various requirements during NIRF dye delivery (18), indicating their flexibility.

In the present study, silica nanoparticles were initially modified to achieve thiol-terminated silica nanoparticles (MB-SHSi) that were employed to encapsulate MB. Subsequently, epidermal growth factor receptor (EGFR), 
a clinically related cancer biomarker that occurs in most epithelial-cell cancers and is a suitable target for cancer detection and treatment (19-21), was conjugated to the MB-SHSi to formulate anti-EGFR/MB-SHSi complexes. It is expected that the anti-EGFR/MB-SHSi complexes may serve as a biocompatible probe to selectively detect lung cancer in a mouse model.

\section{Materials and methods}

Materials. MB, Triton X-100, tetraethyl orthosilicate (TEOS), (3-mercaptopropyl)-trimethoxysilane (MPTMS), bovine serum albumin (BSA), Dulbecco's modified Eagle's medium (DMEM) and MTT were purchased from Sigma-Aldrich (Merck KGaA, Darmstadt, Germany). Anti-EGFR monoclonal antibodies (host mouse; cat. no. sc-80543) were purchased from Santa Cruz Biotechnology Inc., (Santa Cruz, CA, USA). All other regents were of analytical grade and provided by Shanghai Chemical Co., (Shanghai, China).

Cell culture. The human lung carcinoma cell line (A549) was purchased from the Cell Bank of Shanghai Institute of Biochemistry and Cell Biology, Chinese Academy of Sciences (Shanghai, China) and cultured in DMEM supplemented with $10 \%$ fetal bovine serum (FBS; Hyclone; GE Healthcare Bio-Sciences, Pittsburgh, PA, USA), $100 \mathrm{U} / \mathrm{ml}$ penicillin and $100 \mu \mathrm{g} / \mathrm{ml}$ streptomycin in a humidified atmosphere of $95 \%$ air $/ 5 \% \mathrm{CO}_{2}$ in an incubator at $37^{\circ} \mathrm{C}$. All experiments were performed on cells in the logarithmic growth phase.

Animal model. A total of 50 male BALB/c nude mice (age, 5 weeks; weight, 20-22 g) were purchased from Shanghai Laboratory Animal Center (Shanghai, China), housed in a specific-pathogen-free laboratory with a $12 \mathrm{~h}$ light/dark cycle, temperature of $25^{\circ} \mathrm{C}$ and humidity of $50 \%$, and were given free access to food and water. All procedures were conducted in compliance with the institutional and NIH guidelines for the care and use of research animals of The First Affiliated Hospital of Anhui Medical University (Hefei, China). Tumor-bearing mice were produced by inoculating a suspension of A549 cells ( $2 \times 10^{6}$ cells in $0.2 \mathrm{ml}$ physiological saline) subcutaneously into the left flank and used for studies when the tumor volume reached $80-100 \mathrm{~mm}^{3}$. This was calculated via the following formula: Tumor volume: $\left(\mathrm{W}^{2} \mathrm{xL}\right) / 2$, where $\mathrm{W}$ and $\mathrm{L}$ are the shortest and longest diameters, respectively.

Preparation of anti-EGFR/MB-SHSi complexes. MB-SHSi were directly synthesized based on a previous report with minor modifications (18) and using the synchronous hydrolysis of TEOS and MPTMS in a water-in-oil microemulsion. Briefly, a water-in-oil microemulsion containing MB was prepared by mixing $7.5 \mathrm{ml}$ cyclohexane, $1.8 \mathrm{ml}$ Triton $\mathrm{X}-100$, $1.6 \mathrm{ml} \mathrm{n}$-hexanol and $480 \mu \mathrm{l} \mathrm{MB}$ solution $(0.005 \mathrm{~mol} / \mathrm{l})$. After stirring for $30 \mathrm{~min}, 60 \mu \mathrm{l}$ MPTMS and $180 \mu \mathrm{l}$ TEOS were added as precursors for silica matrix formation, followed by the addition of $100 \mu \mathrm{l} \mathrm{NH} \mathrm{NH}_{4} \mathrm{OH}$ to initiate polymerization, and the reaction was left for $24 \mathrm{~h}$ at room temperature. After the reaction was complete, the MB-SHSi nanoparticles were precipitated by the addition of ethanol and washed with water and ethanol several times in order to remove the excess dye molecules and surfactants from the particles. Furthermore, the particle size and zeta potential of MB-SHSi were measured by a Dynamic Light Scattering Analyzer (Brookhaven Instruments Corporation, Holtsville, NY, USA) and a ZetaPlus Zeta Potential Analyzer (Brookhaven Instruments Corporation), respectively. The morphology of the MB-SHSi was further visualized using transmission electron microscopy (JEM-1230; JOEL, Tokyo, Japan).

Anti-EGFR/MB-SHSi complexes were prepared according to the method described by Sokolov et al (22). Briefly, the MB-SHSi were diluted in $20 \mathrm{mM}$ HEPES buffer ( $\mathrm{pH} 7.4)$ to a final concentration of $1 \mathrm{mg} / \mathrm{ml}$. In total, $50 \mu \mathrm{l}$ anti-EGFR monoclonal antibodies (1:500) were diluted in $500 \mu \mathrm{l}$ of the same HEPES buffer. Subsequently, $10 \mathrm{ml}$ of the Au solution prepared above was mixed with the diluted antibody solution for $20 \mathrm{~min}$. A total of $0.5 \mathrm{ml}$ polyethyleneglycol (1\%; molecular weight, 4,000) was added to the mixture to prevent aggregation, and the solution was centrifuged at 7,500 $\mathrm{x} g$ at $25^{\circ} \mathrm{C}$, for $18 \mathrm{~min}$. The anti-EGFR/MB-SHSi complexes were redispersed in PBS buffer ( $\mathrm{pH} 7.4)$ and stored at $4^{\circ} \mathrm{C}$ until subsequent use.

Particle size, zeta potential measurement and morphology observation. The particle size and zeta potential of anti-EGFR/MB-SHSi complexes were measured in triplicate by dynamic light scattering using a Malvern Zetasizer (Nano ZS-90; Malvern Instruments Ltd., Malvern, UK) at $25^{\circ} \mathrm{C}$ with a $90^{\circ}$ scattering angle, according to the manufacturer's instructions. Morphology observation was conducted by transmission electron microscopy (Hitachi, Ltd., Tokyo, Japan) at an accelerating voltage of $80 \mathrm{kV}$, according to the manufacturer's instructions.

\section{Biocompatibility assays}

BSA challenging assay. Anti-EGFR/MB-SHSi complexes were incubated with various BSA solutions ( $\mathrm{pH}$ 7.4) for $1 \mathrm{~h}$ at $37^{\circ} \mathrm{C}$. Alterations in turbidity at $350 \mathrm{~nm}$ were monitored using a spectrophotometer (Hitachi, Ltd.), as reported previously (13).

Hemolysis test. Whole rat blood samples were collected to evaluate the hemolysis of specimens. Furthermore, $0.06 \mathrm{ml}$ anticoagulated rat blood was added to $3 \mathrm{ml}$ of i) $0.9 \% \mathrm{NaCl}$ solution containing different specimens (anti-EGFR/MB-SHSi complexes with various concentrations); ii) PBS [0.01 M (pH 7.4), negative control]; and iii) water (positive control). Subsequently, the contents of the tubes were gently mixed and placed in a water bath at $37^{\circ} \mathrm{C}$. Following incubation for $1 \mathrm{~h}$, the suspension was centrifuged at $2,500 \mathrm{xg}$ at $25^{\circ} \mathrm{C}$, for $10 \mathrm{~min}$ and the absorbance of the supernatant of each tube was measured by ultraviolet spectroscopy (Hitachi, Ltd.) at $545 \mathrm{~nm}$. Samples were run in triplicate (16).

Cytotoxicity assay. The cytotoxicity of anti-EGFR/MB-SHSi complexes was evaluated using the standard MTT assay. Human pulmonary carcinoma A549 cells were seeded at $1.0 \times 10^{4}$ cells/well into 96-well plates and cultured with DMEM medium supplemented with $10 \% \mathrm{FBS}$ at $37^{\circ} \mathrm{C}$ and in a $5 \% \mathrm{CO}_{2}$ environment overnight until they reached $70-80 \%$ confluence. The primary growth medium was replaced by $200 \mu \mathrm{l}$ of fresh 
serum-free DMEM medium, to which anti-EGFR/MB-SHSi complexes were added to achieve various concentrations ranging from 10 to $1,000 \mu \mathrm{g} / \mathrm{ml}$. Plates were then returned to the incubator for another $24 \mathrm{~h}$. After which, $20 \mu \mathrm{l}$ of $5 \mathrm{mg} / \mathrm{ml}$ MTT solution in PBS was added to each well for an additional 4-h incubation. Subsequently, the medium was carefully removed and replaced by $150 \mu \mathrm{l}$ DMSO and measured at $570 \mathrm{~nm}$ using a microplate reader (EL800; BioTek Instruments Inc., Winooski, VT, USA). Untreated cells were used as a control with $100 \%$ viability.

In vivo long-term toxicity. Ordinary BALB/c nude mice were randomly divided into two groups: i) Saline (control) and ii) anti-EGFR/MB-SHSi complexes. Both formulations were administrated once daily over a period of 14 days. Following administration, mice were sacrificed and the main organs were subjected to hematoxylin and eosin (H\&E) staining and observed under an optical microscope (IX51; Olympus Corp., Tokyo, Japan).

In vitro cellular uptake and competitive inhibition experiments. A549 cells were cultured on six-well plates as mentioned. MB-SHSi and anti-EGFR/MB-SHSi complexes were co-incubated with the cells for $2 \mathrm{~h}$. To further determine the EGFR-mediated internalization of anti-EGFR/MB-SHSi complexes, A549 cells were pretreated with excess free antibodies before the addition of anti-EGFR/MB-SHSi complexes. Subsequently, the cells were fixed with $4 \%$ paraformaldehyde for $15 \mathrm{~min}$ and treated with 4',6-diamidino-2-phenylindole (DAPI) for another $15 \mathrm{~min}$ for nucleus staining. Fluorescence images were visualized and captured by confocal laser scanning microscopy (CLSM; Leica TCS SP5; Leica Microsystems $\mathrm{GmbH}$, Wetzlar, Germany) and the fluorescence intensity was quantitatively analyzed by flow cytometry (BD Biosciences, Franklin Lakes, NJ, USA).

In vivo tumor imaging. A549 tumor-bearing nude mice with a tumor volume of $80-100 \mathrm{~mm}^{3}$ [calculated as: Tumor volume: $\left(\mathrm{L} \mathrm{x} \mathrm{W}^{2}\right) / 2$ ] were administered intravenously with MB-SHSi and anti-EGFR/MB-SHSi complexes in order to verify the tumor target ability of different nanoparticles. In vivo imaging and tumor target ability efficacy of nanoparticles were evaluated using an in vivo imaging system (FXPRO; Kodak, Rochester, NY, USA) equipped with NIR filter sets (excitation/emission, 680/790 nm).

Statistical analysis. Data are presented as the mean \pm standard deviation. Statistical significance was assessed using a two-tailed Student's t-test or one-way analysis of variance using Origin 8.0 (OriginLab Corporation, Northampton, MA, USA). $\mathrm{P}<0.01$ was considered to indicate a statistically significant difference.

\section{Results and Discussion}

Preparation of anti-EGFR/MB-SHSi complexes. The reverse water-in-oil micro-emulsion containing innumerable nanosized water pools is an ideal place for a diameter-controlled reaction (23). When the MB-SHSi were formed, MB was incorporated into MB-SHSi by means of physical absorption
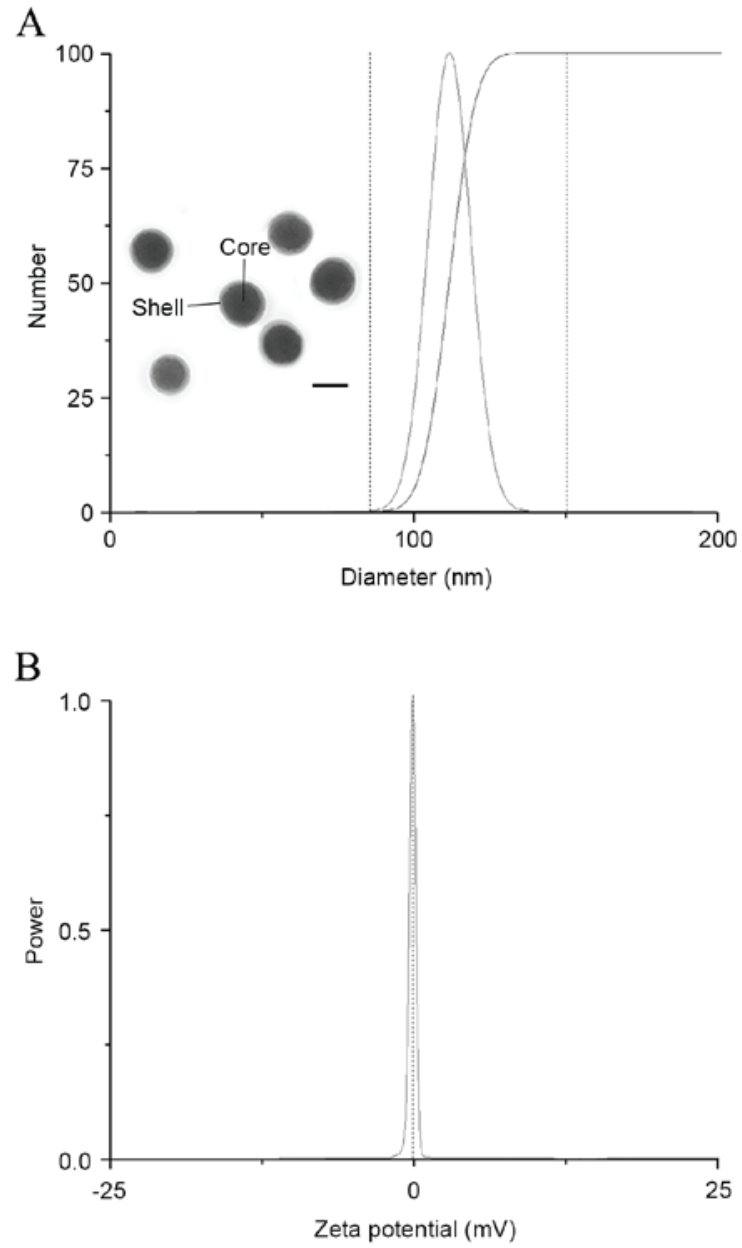

Figure 1. (A) Particle size, morphology and (B) zeta potential of anti-EGFR/MB-SHSi complexes. Scale bar, $100 \mu \mathrm{m}$. EGFR, epidermal growth factor receptor; MB-SHSi, MB-encapsulated thiol-terminated silica nanoparticles.

and/or chemical embedment to formulate MB-SHSi. After the formation of MB-SHSi, anti-EGFR monoclonal antibodies were added and the thiol groups of anti-EGFR monoclonal antibodies were expected to form a disulfide bond with MB-SHSi and construct anti-EGFR/MB-SHSi complexes.

Particle size, zeta potential measurement and morphology observation. Particle size and zeta potential are two of the most important parameters that affect the performance of nanoparticles. As a result, an ideal delivery system is required to be carefully tuned in order to achieve the optimal therapeutic effect in cancer treatment. Multiple studies have demonstrated that the biodistribution behavior and cellular uptake efficiency of nanoparticles are relevant to their particle size and zeta potential $(24,25)$. In general, a smaller size usually leads to preferable cellular uptake and a superior therapeutic effect of particles, as they can be readily recognized and transported by the corresponding receptor or channel (26). As displayed in Fig. 1, anti-EGFR/MB-SHSi complexes had a nanoscale size of $\sim 100 \mathrm{~nm}$ with a neutral surface charge. Transmission electron microscopy images further demonstrated that anti-EGFR/MB-SHSi complexes were spherical in shape and had a compacted structure with good dispersity. 

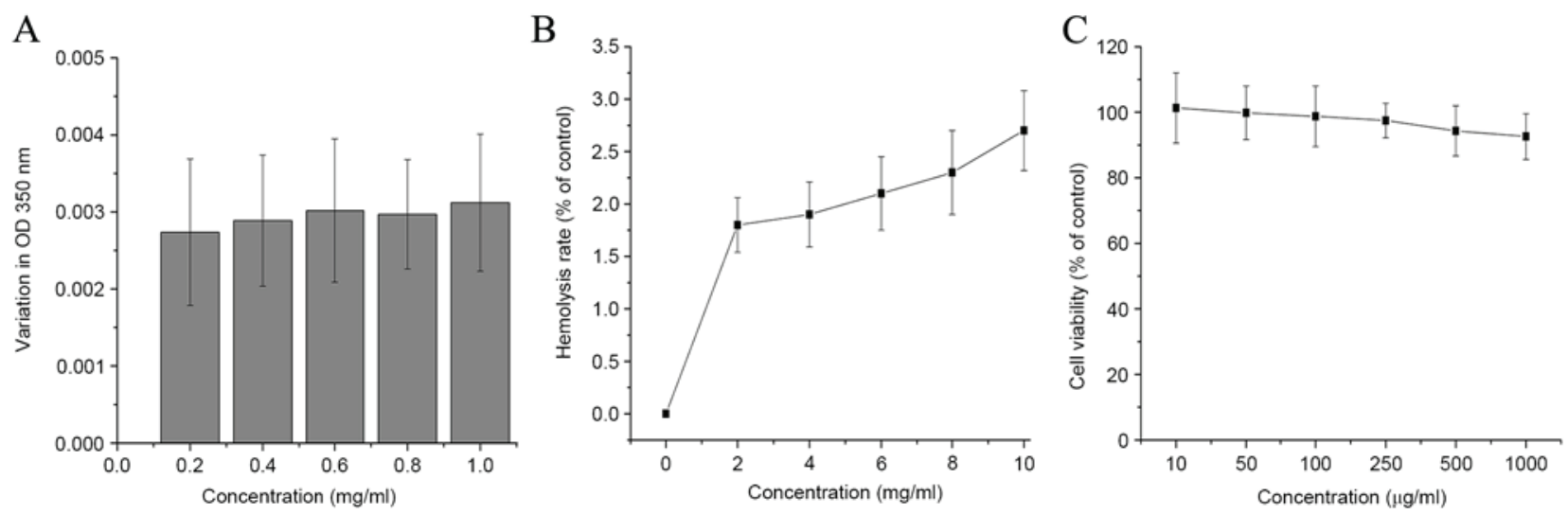

Figure 2. (A) Bovine serum albumin challenging assay, (B) hemolysis test and (C) cytotoxicity assay of anti-EGFR/MB-SHSi complexes. Data were expressed as mean \pm standard deviation $(\mathrm{n}=5$ ). EGFR, epidermal growth factor receptor; MB-SHSi, MB-encapsulated thiol-terminated silica nanoparticles; OD, optical density.
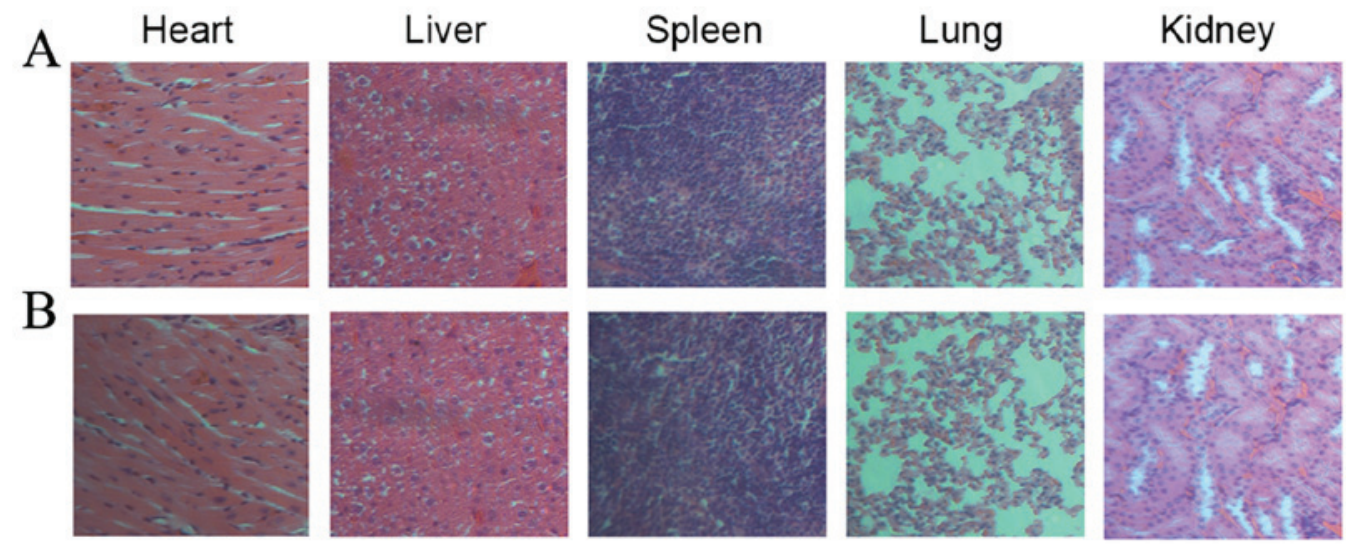

Figure 3. Hematoxylin and eosin staining images (magnification, x200) of main organs from mice administered with (A) saline and (B) anti-EGFR/MB-SHSi complexes. EGFR, epidermal growth factor receptor; MB-SHSi, MB-encapsulated thiol-terminated silica nanoparticles.

Biocompatibility assays. The application of a probe for cancer detection necessitates safety profiles, including BSA challenge, hemolysis and cytotoxicity. The circulatory system comprises many negatively charged serum proteins (such as albumin) that may be absorbed into nanoparticles and induce immediate toxicity. In the present study, BSA was used to mimic the negatively charged serum proteins to evaluate the stability of anti-EGFR/MB-SHSi complexes. As depicted in Fig. 2A, anti-EGFR/MB-SHSi complexes exhibited a low absorption value with negligible turbidity change as the BSA concentrations increased, as evidenced by the invariant $\mathrm{OD}_{350 \mathrm{~nm}}$. Therefore, the anti-EGFR/MB-SHSi complexes did not display significant hemolytic activity at all concentrations tested $(<3 \%)$, which indicated that anti-EGFR/MB-SHSi complexes would be non-toxic towards erythrocytes after intravenous administration. The hemolysis test was also conducted to verify the safety profiles of anti-EGFR/MB-SHSi complexes (Fig. 2B). The results obtained from the cytotoxicity assay in Fig. 2C demonstrated that A549 cells treated with various concentrations of anti-EGFR/MB-SHSi complexes, even at the highest one of $1,000 \mu \mathrm{g} / \mathrm{ml}$, yielded a similar viability to the control (untreated cells). In the long-term toxicity assay, after 14 days of continuous injection of anti-EGFR/MB-SHSi complexes, the main organs of mice were excised for H\&E staining.
As displayed in Fig. 3 and compared to the saline group, all the organs from anti-EGFR/MB-SHSi complexes were normal and without any pathological changes. Overall, the above results provided decisive evidence that anti-EGFR/MB-SHSi complexes may be safely used as a probe that can be employed for in vivo application.

In vitro cellular uptake and competitive inhibition experiments. As shown in Fig. 4A, CLSM observations at $2 \mathrm{~h}$ indicated red fluorescence signals in the cytoplasm of A549 cells (blue signal from DAPI indicated the location of nucleus), which was attributed to MB, suggesting that both MB-SHSi and anti-EGFR/MB-SHSi complexes successfully entered the cells. Notably, the fluorescence signal gradually became stronger as the incubation time increased, indicating that the intracellular uptake of the nanoparticles increased in a time-dependent manner. However, under the same conditions, anti-EGFR/MB-SHSi complexes exhibited a higher intensity when compared with MB-SHSi.

Flow cytometry was used to quantitatively analyze intracellular uptake, as presented in Fig. 4B. The fluorescence intensity of anti-EGFR/MB-SHSi complexes was $\sim 5.74$-fold higher than that of MB-SHSi after incubation for $2 \mathrm{~h}$. In a competitive experiment, A549 cells were treated with free 

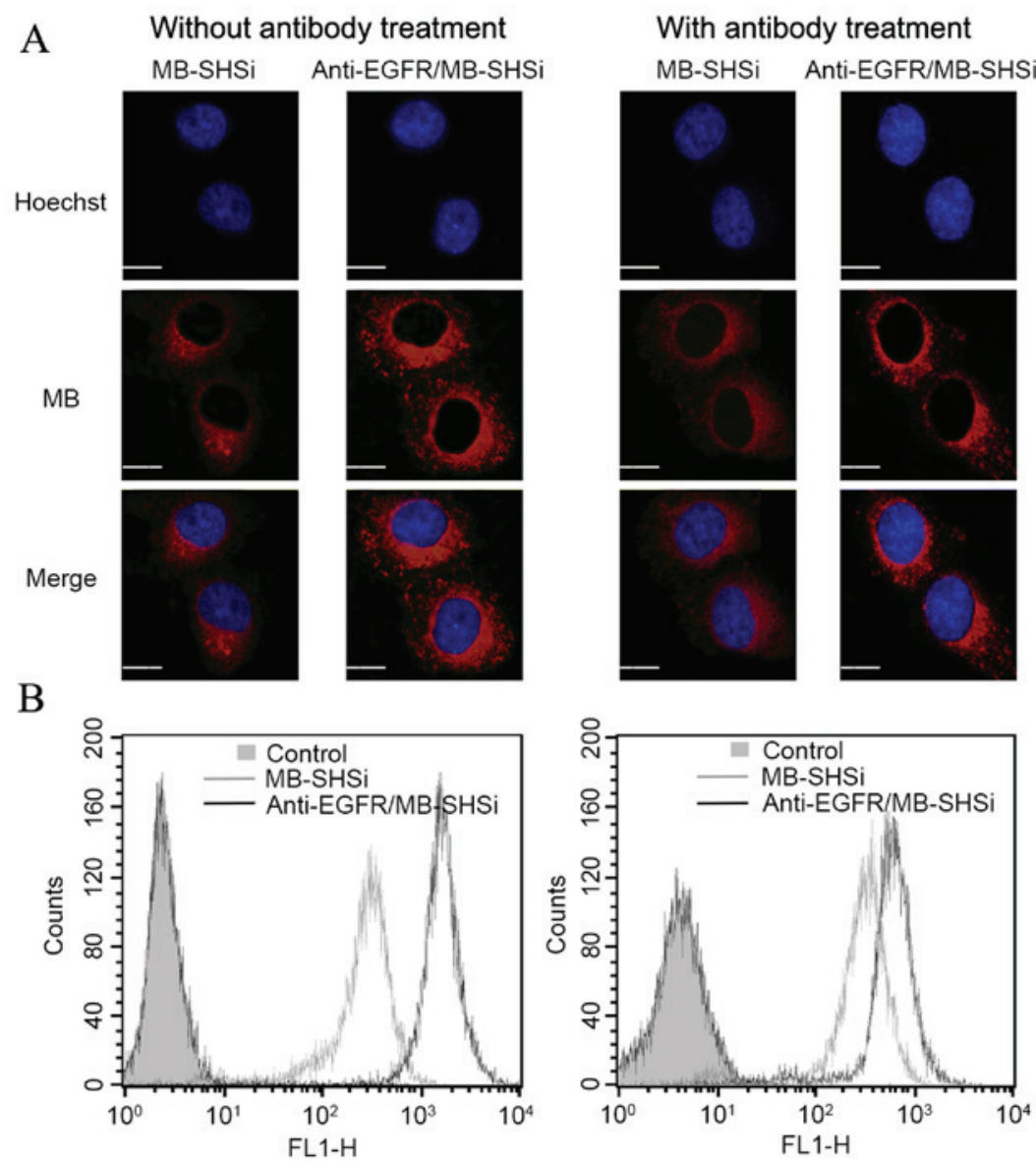

Figure 4. (A) Confocal laser scanning microscopy images of the cellular uptake profile of MB-SHSi and anti-EGFR/MB-SHSi complexes pretreated with or without antibody at $2 \mathrm{~h}$ post incubation. (B) Representative flow cytometry analysis of MB-SHSi and anti-EGFR/MB-SHSi complexes pretreated with or without antibody at $2 \mathrm{~h}$ post incubation. Scale bar, $20 \mu \mathrm{m}$. EGFR, epidermal growth factor receptor; MB-SHSi, MB-encapsulated thiol-terminated silica nanoparticles.
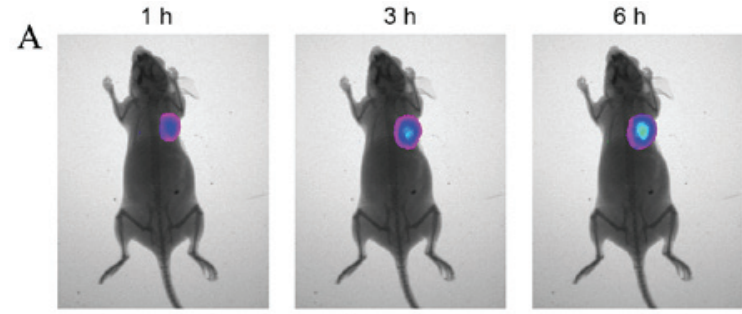

B

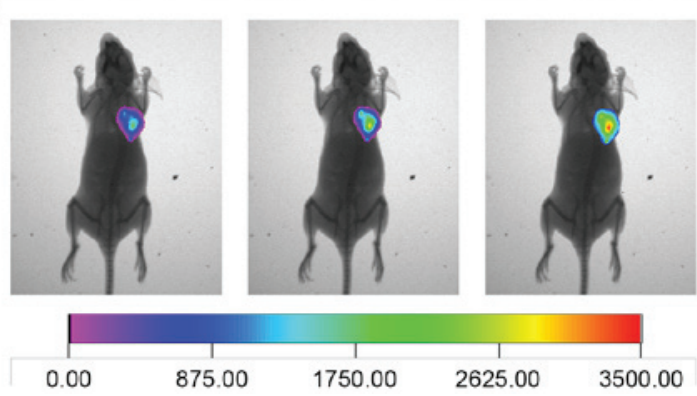

Figure 5. In vivo real-time imaging of (A) MB-SHSi and (B) anti-EGFR/ MB-SHSi complexes at different time intervals. EGFR, epidermal growth factor receptor; MB-SHSi, MB-encapsulated thiol-terminated silica nanoparticles.

antibodies prior to incubation with the nanoparticles in order to investigate the mechanism of internalization. Fluorescence microscopy and flow cytometry demonstrated a decrease in the fluorescence intensity in tumor cells pretreated with antibodies compared with the untreated cells. This result indicates that the free antibodies were able to competitively bind to EGFR receptors and block the binding of anti-EGFR/MB-SHSi complexes to these receptors, which confirmed that a receptor-mediated pathway was utilized to traffic the nanoparticles into cells.

In vivo tumor imaging. The in vivo biodistribution of anti-EGFR/MB-SHSi complexes in tumor-bearing nude mice was investigated using a non-invasive real-time in vivo imaging technique to test the potential utility of anti-EGFR/MB-SHSi complexes as a probe in detecting A549 lung cancer. As displayed in Fig. 5, compared with MB-SHSi, at $1 \mathrm{~h}$ post injection, anti-EGFR/MB-SHSi complexes were rapidly accumulated at the tumor site. Higher accumulation and retention of anti-EGFR/MB-SHSi complexes within the tumor region were detected at $3 \mathrm{~h}$ post injection. At $6 \mathrm{~h}$, the anti-EGFR/MB-SHSi complexes were clearly retained in the tumor site with a further increase in fluorescence intensity, while the fluorescence intensity in the MB-SHSi group was marginally stronger than that of the anti-EGFR/MB-SHSi complexes at $1 \mathrm{~h}$. The superior tumor target ability of anti-EGFR/MB-SHSi complexes might be ascribed to a combination of an EPR effect (23) 
and EGFR-mediated uptake mechanism. Furthermore, these observations provided decisive evidence that the anti-EGFR/MB-SHSi complexes designed in the present study were suitable as a tumor-specific probe for cancer detection.

In conclusion, a well-designed nanosystem using anti-EGFR/MB-SHSi complexes was successfully developed in the present study as a probe for cancer detection. The anti-EGFR/MB-SHSi complexes, which had a diameter of $\sim 100 \mathrm{~nm}$ and a neutral surface charge, demonstrated low protein absorption and hemolytic activity in vitro with negligible cytotoxicity both in vitro and in vivo. Overall, the anti-EGFR/MB-SHSi complexes exhibited EGFR-mediated cellular uptake behavior, which contributes to its specific tumor-targeting profile in vivo. Therefore, anti-EGFR/MB-SHSi complexes with both high biocompatibility and target ability may be employed as a potential probe for in vivo cancer detection.

\section{Acknowledgements}

The present study was supported by the National Natural Science Foundation of China (grant no. 81302028).

\section{References}

1. Jemal A, Thun MJ, Ries LA, Howe HL, Weir HK, Center MM, Ward E, Wu XC, Eheman C, Anderson R, et al: Annual report to the nation on the status of cancer, 1975-2005, featuring trends in lung cancer, tobacco use, and tobacco control. J Natl Cancer Inst 100: 1672-1694, 2008.

2. Qu MH, Zeng RF, Fang S, Dai QS, Li HP and Long JT: Liposome-based co-delivery of siRNA and docetaxel for the synergistic treatment of lung cancer. Int J Pharm 474: 112-122, 2014.

3. Greenlee RT, Murray T, Bolden S and Wingo PA: Cancer statistics, 2000. CA Cancer J Clin 50: 7-33, 2000.

4. Ballou B, Ernst LA and Waggoner AS: Fluorescence imaging of tumors in vivo. Curr Med Chem 12: 795-805, 2005.

5. He X, Wang $\mathrm{K}$ and Cheng Z: In vivo near-infrared fluorescence imaging of cancer with nanoparticle-based probes. Wiley Interdiscip Rev Nanomed Nanobiotechnol 2: 349-366, 2010.

6. Jain KK: Nanotechnology in clinical laboratory diagnostics. Clin Chim Acta 358: 37-54, 2005.

7. Nie S, Xing Y, Kim GJ and Simons JW: Nanotechnology applications in cancer. Annu Rev Biomed Eng 9: 257-288, 2007.

8. Burns AA, Vider J, Ow H, Herz E, Penate-Medina O, Baumgart M, Larson SM, Wiesner U and Bradbury M: Fluorescent silica nanoparticles with efficient urinary excretion for nanomedicine. Nano Lett 9: 442-448, 2008.

9. Lee S, Ryu JH, Park K, Lee A, Lee SY, Youn IC, Ahn CH, Yoon SM, Myung SJ, Moon DH, et al: Polymeric nanoparticle-based activatable near-infrared nanosensor for protease determination in vivo. Nano Lett 9: 4412-4416, 2009.

10. Li S, Goins B, Zhang L and Bao A: Novel multifunctional theranostic liposome drug delivery system: Construction, characterization, and multimodality MR, near-infrared fluorescent and nuclear imaging. Bioconjug Chem 23: 1322-1332, 2012.
11. Park K, Lee S, Kang E, Kim K, Choi K and Kwon IC: New generation of multifunctional nanoparticles for cancer imaging and therapy. Adv Funct Mater 19: 1553-1566, 2009.

12. Santra S, Kaittanis C, Grimm J and Perez JM: Drug/dye-loaded, multifunctional iron oxide nanoparticles for combined targeted cancer therapy and dual optical/magnetic resonance imaging. Small 5: 1862-1868, 2009.

13. Wang C, Bao X, Ding X, Ding Y, Abbad S, Wang Y, Li M, Su Y, Wang W and Zhou J: A multifunctional self-dissociative polyethyleneimine derivative coating polymer for enhancing the gene transfection efficiency of DNA/polyethyleneimine polyplexes in vitro and in vivo. Polym Chem 6: 780-796, 2015.

14. Abbad S, Wang C, Waddad AY, Lv H and Zhou J: Preparation, in vitro and in vivo evaluation of polymeric nanoparticles based on hyaluronic acid-poly (butyl cyanoacrylate) and D-alpha-tocopheryl polyethylene glycol 1000 succinate for tumor-targeted delivery of morin hydrate. Int J Nanomedicine 10: 305-320, 2015.

15. Deng T, Li JS, Jiang JH, Shen GL and Yu RQ: Preparation of near-IR fluorescent nanoparticles for fluorescence-anisotropy-based immunoagglutination assay in whole blood. Adva Funct Mater 16: 2147-2155, 2006.

16. Lu H, Zhang H, Zhang D, Lu H and Ma D: A biocompatible reconstituted high-density lipoprotein nano-system as a probe for lung cancer detection. Med Sci Monit 21: 2726-2733, 2015.

17. Lee SJ, Park K, Oh YK, Kwon SH, Her S, Kim IS, Choi K, Lee SJ, Kim H, Lee SG, et al: Tumor specificity and therapeutic efficacy of photosensitizer-encapsulated glycol chitosan-based nanoparticles in tumor-bearing mice. Biomaterials 30: 2929-2939, 2009.

18. He X, Wu X, Wang K, Shi B and Hai L: Methylene blue-encapsulated phosphonate-terminated silica nanoparticles for simultaneous in vivo imaging and photodynamic therapy. Biomaterials 30: 5601-5609, 2009.

19. Ciardiello F and Tortora G: EGFR antagonists in cancer treatment. N Engl J Med 358: 1160-1174, 2008.

20. El-Sayed IH, Huang X and El-Sayed MA: Surface plasmon resonance scattering and absorption of anti-EGFR antibody conjugated gold nanoparticles in cancer diagnostics: Applications in oral cancer. Nano Lett 5: 829-834, 2005.

21. Nicholson RI, Gee JM and Harper ME: EGFR and cancer prognosis. Eur J Cancer 37 (Suppl 4): S9-S15, 2001.

22. Sokolov K, Follen M, Aaron J, Pavlova I, Malpica A, Lotan R and Richards-Kortum R: Real-time vital optical imaging of precancer using anti-epidermal growth factor receptor antibodies conjugated to gold nanoparticles. Cancer Res 63: 1999-2004, 2003.

23. Wang C, Chen S, Yu Q, Hu F and Yuan H: Taking advantage of the disadvantage: Employing the high aqueous instability of amorphous calcium carbonate to realize burst drug release within cancer cells. J Mater Chem B 5: 2068-2073, 2017.

24. He C, Hu Y, Yin L, Tang C and Yin C: Effects of particle size and surface charge on cellular uptake and biodistribution of polymeric nanoparticles. Biomaterials 31: 3657-3666, 2010.

25. Limbach LK, Li Y, Grass RN, Brunner TJ, Hintermann MA, Muller M, Gunther D and Stark WJ: Oxide nanoparticle uptake in human lung fibroblasts: Effects of particle size, agglomeration, and diffusion at low concentrations. Environ Sci Technol 39: 9370-9376, 2005.

26. Wang C, Li M, Yang T, Ding X, Bao X, Ding Y, Xiong H, Wu Y, Wang $\mathrm{W}$ and Zhou J: A self-assembled system for tumor-targeted co-delivery of drug and gene. Mater Sci Eng C Mater Biol Appl 56: 280-285, 2015. 